PROCEEDINGS OF THE

AMERICAN MATHEMATICAL SOCIETY

Volume 133, Number 7, Pages 2045-2050

S 0002-9939(05)07721-X

Article electronically published on January 21, 2005

\title{
UNIFORM EMBEDDINGS OF BOUNDED GEOMETRY SPACES INTO REFLEXIVE BANACH SPACE
}

\author{
NATHANIAL BROWN AND ERIK GUENTNER
}

(Communicated by Joseph A. Ball)

\begin{abstract}
We show that every metric space with bounded geometry uniformly embeds into a direct sum of $l^{p}(\mathbb{N})$ spaces ( $p$ 's going off to infinity). In particular, every sequence of expanding graphs uniformly embeds into such a reflexive Banach space even though no such sequence uniformly embeds into a fixed $l^{p}(\mathbb{N})$ space. In the case of discrete groups we prove the analogue of a- $T$-menability - the existence of a metrically proper affine isometric action on a direct sum of $l^{p}(\mathbb{N})$ spaces.
\end{abstract}

\section{INTRODUCTION}

Gromov introduced the notion of uniform embeddability and later suggested that uniform embeddability of a discrete group into a Hilbert space, or even into a uniformly convex Banach space, might be relevant for applications to the Novikov conjecture [3, 2, Yu subsequently proved the Coarse Baum-Connes and Novikov conjectures for groups which are uniformly embeddable into a Hilbert space 15 13. This led to the verification of the Novikov conjecture for large classes of discrete groups, including, for example, linear groups [6] and, more generally, exact groups [12, 7].

The question of whether every discrete group might be uniformly embeddable in Hilbert space remained open for some time. Gromov ended the speculation by announcing the construction of a discrete group which cannot be uniformly embedded into Hilbert space [5, 4]. Nevertheless, the Novikov conjecture remains valid for the groups Gromov constructs.

Two natural questions arise: (1) Can one prove the Novikov conjecture for discrete groups which uniformly embed into other nice Banach spaces? (2) Does every discrete group embed into a nice Banach space? How 'nice' the range Banach space needs to be (for question (1)) is not yet clear, although quite recently Kasparov and $\mathrm{Yu}$ have achieved exciting results in this direction [9]. In this note we show that every metric space with bounded geometry can be uniformly embedded into a fairly nice Banach space. More precisely, our main result is the following:

Theorem 1. Let $X$ be a metric space with bounded geometry. There exists a sequence of positive real numbers $\left\{p_{n}\right\}$ and a uniform embedding of $X$ into the $l^{2}$-direct sum $\bigoplus l^{p_{n}}(\mathbb{N})$.

Received by the editors September 17, 2003 and, in revised form, March 5, 2004.

2000 Mathematics Subject Classification. Primary 46B07.

The authors were partially supported by grants from the U.S. National Science Foundation.

(C) 2005 American Mathematical Society Reverts to public domain 28 years from publication 2045 
The Banach space in the statement is reflexive. Thus, every bounded geometry metric space can be uniformly embedded into a reflexive Banach space. In particular, every sequence of expanding graphs can be uniformly embedded into a reflexive Banach space, answering a question pondered by several experts. As observed by Gromov, a sequence of expanding graphs cannot be uniformly embedded into Hilbert space [4] (see [8] for a proof); less well-known observations by Roe and, respectively, Ozawa are that such a sequence cannot be uniformly embedded into $l^{p}(\mathbb{N})$ for any finite $p$ (cf. [10]), or any other Banach space with an unconditional basis and finite co-type [11. Hence the result above seems to give a uniform embedding into the simplest kind of Banach space that is capable of containing all bounded geometry metric spaces.

In the case of discrete groups we prove the following stronger result.

Theorem 2. Let $\Gamma$ be a countable discrete group. There exists a sequence of positive real numbers $\left\{p_{n}\right\}$ and a metrically proper affine isometric action of $\Gamma$ on the $l^{2}$ direct sum $\bigoplus l^{p_{n}}(\mathbb{N})$. In particular, there exists a uniform embedding $\Phi: \Gamma \rightarrow$ $\bigoplus l^{p_{n}}(\mathbb{N})$ such that $\|\Phi(s)-\Phi(t)\|=\left\|\Phi\left(t^{-1} s\right)\right\|$, for all $s, t \in \Gamma$.

This theorem is a version of a- $T$-menability involving a reflexive Banach space in place of a Hilbert space. The remarks above apply equally to the groups constructed by Gromov: they cannot be uniformly embedded into, and in particular do not admit metrically proper affine isometric actions on, $l^{p}(\mathbb{N})$ for any finite $p$. Thus, again, the result is in some sense optimal.

The proofs of the two results above have certain similarities though the actual constructions involved are slightly different. In the next section we collect several facts needed for both proofs and set our notation. In the remaining two sections we construct the required embedding and affine action, proving the theorems.

\section{Notation, DEFINITIONS AND BASIC FACTS}

For a countable set $S$ let $l^{p}(S)$ denote the usual sequence space and $\|\cdot\|_{p}$ the corresponding norm (we allow $1 \leq p \leq \infty$ ). For a sequence $\mathcal{E}_{n}$ of Banach spaces let $\bigoplus \mathcal{E}_{n}$ denote the $l^{2}$-direct sum defined by $\bigoplus \mathcal{E}_{n}=\left\{\left(z_{n}\right): \sum\left\|z_{n}\right\|^{2}<\infty\right\}$, with the obvious norm. Since the dual space of an $l^{2}$-direct sum is the $l^{2}$-direct sum of the dual spaces we see that if every $\mathcal{E}_{n}$ is reflexive, then so is $\bigoplus \mathcal{E}_{n}$. In particular, for every sequence of real numbers $\left\{p_{n}\right\}, \bigoplus l^{p_{n}}(\mathbb{N})$ is a reflexive Banach space.

A metric space $X$ is locally finite if every ball is finite; it has bounded geometry if for all $R>0$ there exists $C>0$ such that every $R$-ball in $X$ contains at most $C$ points. With these conventions a bounded geometry metric space is necessarily discrete and countable.

A function $\Phi: X \rightarrow \mathcal{E}$ from a metric space $X$ to a Banach space $\mathcal{E}$ is a uniform embedding if there exist non-decreasing proper functions $\rho_{ \pm}:[0, \infty) \rightarrow \mathbb{R}$ such that

$$
\rho_{-}(d(x, y)) \leq\|\Phi(x)-\Phi(y)\| \leq \rho_{+}(d(x, y)), \quad \text { for all } x, y \in X,
$$

where $\|\cdot\|$ denotes the norm in $\mathcal{E}$.

We require the following elementary fact.

Lemma 3. Let $S$ be a set, and let $\alpha \geq 0$ and $\beta \geq 1$. Then $\|f\|_{p} \rightarrow\|f\|_{\infty}$ as $p \rightarrow \infty$ uniformly on sets of the form

$$
\mathcal{S}_{\alpha, \beta}=\left\{f \in l^{\infty}(S):\|f\|_{\infty} \leq \alpha \text { and } \# \operatorname{supp}(f) \leq \beta\right\},
$$

where \# $\operatorname{supp}(f)$ denotes the cardinality of the support of $f$. 
Proof. The following inequalities imply the lemma:

$$
\begin{aligned}
\|f\|_{\infty} & \leq\|f\|_{p}=\left\{\sum_{s \in \operatorname{supp}(f)}|f(s)|^{p}\right\}^{1 / p} \\
& \leq\|f\|_{\infty}\left\{\sum_{s \in \operatorname{supp}(f)} 1\right\}^{1 / p} \leq\|f\|_{\infty} \beta^{1 / p} .
\end{aligned}
$$

\section{Bounded GeOMETRY CASE}

In this section we prove Theorem 1 The main observation is that every locally finite metric space enjoys a weak analogue of Yu's property A 15. More precisely we have the following lemma.

Lemma 4. Let $X$ be a locally finite metric space. Then there exists a sequence of mappings $\phi^{n}: X \rightarrow l^{\infty}(X), x \mapsto \phi_{x}^{n}$ such that

(1) $\left\|\phi_{x}^{n}\right\|_{\infty}=1$, for all $x \in X$ and $n \in \mathbb{N}$;

(2) $\operatorname{supp}\left(\phi_{x}^{n}\right) \subset B_{n}(x)$, where $B_{n}(x)$ is the ball of radius $n$ about $x$;

(3) $\left\|\phi_{x}^{n}-\phi_{y}^{n}\right\|_{\infty} \leq d(x, y) / n$, for all $x, y \in X$.

Proof. Define functions $\phi^{n}: X \rightarrow l^{\infty}(X)$ as follows:

$$
\phi_{x}^{n}(y)= \begin{cases}1-\frac{d(x, y)}{n}, & d(x, y) \leq n, \\ 0, & d(x, y) \geq n .\end{cases}
$$

Clearly conditions (1) and (2) are satisfied. Condition (3) is easily checked by considering cases.

Case $z \notin B_{n}(x) \cup B_{n}(y): \phi_{x}^{n}(z)=0$ and $\phi_{y}^{n}(z)=0$.

$\overline{\text { Case } z \in B_{n}(x) \cap B_{n}(y):}$ By the triangle inequality,

$$
\left|\phi_{x}^{n}(z)-\phi_{y}^{n}(z)\right|=|d(x, z)-d(y, z)| / n \leq d(x, y) / n .
$$

Case $z \in B_{n}(x)-B_{n}(y):$ Again by the triangle inequality,

$$
\left|\phi_{x}^{n}(z)-\phi_{y}^{n}(z)\right|=(n-d(x, z)) / n \leq(d(y, z)-d(x, z)) / n \leq d(x, y) / n .
$$

The remaining case is similar.

The conditions (1)-(3) in the lemma are indeed a weak form of Property A. For a bounded geometry space $X, \mathrm{Tu} 14$ showed that Property A is equivalent to the existence of a sequence of functions $\psi^{n}: X \rightarrow l^{2}(X)$ satisfying the following conditions:

(a) $\left\|\psi_{x}^{n}\right\|_{2}=1, \forall x \in X$ and $\forall n \in \mathbb{N}$;

(b) $\forall n \in \mathbb{N} \exists R>0$ such that $\operatorname{supp}\left(\psi_{x}^{n}\right) \subset B_{R}(x)$;

(c) $\forall C>0\left\|\psi_{x}^{n}-\psi_{y}^{n}\right\|_{2} \rightarrow 0$ uniformly for $x, y \in X$ satisfying $d(x, y) \leq C$.

We note that conditions (2) and (3) in the lemma could be replaced by direct analogues of conditions (b) and (c) (for example, replace the precise estimate in (3) by a statement involving only uniform convergence as in (c)) at the cost of complicating some of the arguments below. 
Proof of Theorem 1. Since $X$ is assumed to be of bounded geometry, the cardinalities of the supports of the functions $\phi_{x}^{n}$ (defined as in the previous lemma) are uniformly bounded above for each $n$ (independent of $x$ ). Hence for each integer $n$ we can, by Lemma 3 find a real number $p_{n}$ such that

$$
\left\|\phi_{x}^{n}-\phi_{y}^{n}\right\|_{p_{n}} \leq\left\|\phi_{x}^{n}-\phi_{y}^{n}\right\|_{\infty}+1 / n,
$$

for all $x, y \in X$. Combined with condition (3) of the lemma this implies, in particular, that

$$
\left\|\phi_{x}^{n}-\phi_{y}^{n}\right\|_{p_{n}} \leq \frac{d(x, y)+1}{n}
$$

for all $n \in \mathbb{N}$ and $x, y \in X$.

Now fix a base point $x_{0} \in X$ and define, for all $x \in X$,

$$
\Phi(x)=\bigoplus\left(\phi_{x}^{n}-\phi_{x_{0}}^{n}\right) .
$$

A simple calculation reveals that

$$
\|\Phi(x)-\Phi(y)\|^{2}=\sum_{n \in \mathbb{N}}\left\|\phi_{x}^{n}-\phi_{y}^{n}\right\|_{p_{n}}^{2} \leq(d(x, y)+1)^{2} \sum_{n \in \mathbb{N}} \frac{1}{n^{2}} .
$$

Setting $y=x_{0}$ and observing that $\Phi\left(x_{0}\right)=0$ we conclude that indeed $\Phi(x) \in$ $\bigoplus l^{p_{n}}(X)$ for all $x \in X$. It remains to see that $\Phi: X \rightarrow \bigoplus l^{p_{n}}(X)$ is a uniform embedding. Consulting the previous inequality once again we see that we may define $\rho_{+}(r)=\left(\sum_{n \in \mathbb{N}} \frac{1}{n^{2}}\right)^{1 / 2}(r+1)$.

We define a lower distortion function by

$$
\rho_{-}(r)=\inf _{d(x, y) \geq r}\|\Phi(x)-\Phi(y)\|
$$

and are left to check that $\rho_{-}(r) \rightarrow \infty$ as $r \rightarrow \infty$. In other words, for every integer $R>0$ we must find an $r_{0}$ such that $\|\Phi(x)-\Phi(y)\|>R$ for all $x, y \in X$ satisfying $d(x, y)>r_{0}$. This is easy to do: since each $\phi_{x}^{n}$ is supported in the ball of radius $n$ about $x$, if $d(x, y)>2 R^{2}$, it follows that

$$
\|\Phi(x)-\Phi(y)\|^{2}>\sum_{n=1}^{R^{2}}\left\|\phi_{x}^{n}-\phi_{y}^{n}\right\|_{p_{n}}^{2} \geq \sum_{n=1}^{R^{2}}\left\|\phi_{x}^{n}-\phi_{y}^{n}\right\|_{\infty}^{2}=R^{2} .
$$

This concludes the proof.

\section{Discrete Group CASE}

In this section we prove Theorem 2. The proof is similar to one of the standard proofs that amenable groups are uniformly embeddable into Hilbert space [1].

Let $\Gamma$ be a countable discrete group. Equip $\Gamma$ with a proper length function; if $\Gamma$ is finitely generated, this could be a word length function. The length of an element $s \in \Gamma$ is denoted $|s|$.

The left regular representation of $\Gamma$ on $l^{p}(\Gamma)$ is denoted $f \longmapsto s \cdot f$; these are isometric actions. We regard $l^{\infty}(\Gamma) \subset \mathcal{B}\left(l^{2}(\Gamma)\right)$ as multiplication operators; we regard $\Gamma \subset \mathcal{B}\left(l^{2}(\Gamma)\right)$ via the left regular representation. With these conventions we have $s f s^{*}=s \cdot f$ for all $s \in \Gamma$ and $f \in l^{\infty}(\Gamma)$.

Lemma 5. For every $C>0$ and $\varepsilon>0$ there exists a non-negative, finitely supported function $f$ on $\Gamma$ such that $\|f\|_{\infty}=f(e)=1$ and $\|s \cdot f-f\|_{\infty}<\varepsilon$, for all $s \in \Gamma$ with $|s| \leq C$. 
Proof. Consider the $C^{*}$-algebra $B=C_{r}^{*}(\Gamma)+\mathcal{K}\left(l^{2}(\Gamma)\right) \subset B\left(l^{2}(\Gamma)\right)$. Define $h \in c_{0}(\Gamma)$ by $h(s)=|s|^{-1}($ set $h(e)=1)$. Observe that $h \in c_{0}(\Gamma) \subset l^{\infty}(\Gamma) \cap \mathcal{K}\left(l^{2}(\Gamma)\right)$ is a strictly positive element in $\mathcal{K}\left(l^{2}(\Gamma)\right)$. Consequently, $\left\{h^{1 / n}\right\}$ is an approximate unit for $\mathcal{K}\left(l^{2}(\Gamma)\right)$ and the convex hull of $\left\{h^{1 / n}\right\}$ contains an approximate unit for $\mathcal{K}\left(l^{2}(\Gamma)\right)$ which is quasicentral in $B$. In particular, given $C>0$ and $\varepsilon>0$ there exists a convex combination $g$ of the $\left\{h^{1 / n}\right\}$ such that

$$
\|s \cdot g-g\|_{\infty}=\left\|s g s^{*}-g\right\|=\|s g-g s\|<\frac{\varepsilon}{3},
$$

for all $s \in \Gamma$ with $|s| \leq C$. Note that $g \in c_{0}(\Gamma)$ satisfies $\|g\|_{\infty}=g(e)=1$, and let $f$ be a function of finite support such that $\|f-g\|_{\infty}<\varepsilon / 3,\|f\|_{\infty}=f(e)=1$. The proof concludes with a simple estimate:

$$
\|s \cdot f-f\|_{\infty} \leq\|s \cdot f-s \cdot g\|_{\infty}+\|s \cdot g-g\|_{\infty}+\|g-f\|_{\infty}<\varepsilon,
$$

for all $s \in \Gamma$ with $|s| \leq C$.

Proof of Theorem 2. Apply the previous lemma to a sequence of $C$ 's tending to infinity and a sequence of $\varepsilon$ 's tending sufficiently fast to zero to obtain a sequence of finitely supported functions $\left\{f_{n}\right\}$ such that

$$
\sum_{n=1}^{\infty}\left\|s \cdot f_{n}-f_{n}\right\|_{\infty}^{2}<\infty
$$

for all $s \in \Gamma$. Apply Lemma 3 to obtain a sequence $\left\{p_{n}\right\}$ such that

$$
\sum_{n=1}^{\infty}\left\|s \cdot f_{n}-f_{n}\right\|_{p_{n}}^{2}<\infty
$$

for all $s \in \Gamma$. By virtue of this inequality we may define $\Phi: \Gamma \rightarrow \bigoplus l^{p_{n}}(\Gamma)$ by $\Phi(s)=\bigoplus\left(s \cdot f_{n}-f_{n}\right)$.

Denote by $\lambda$ the sum of the left regular representations of $\Gamma$ on $\bigoplus l^{p_{n}}(\Gamma) ; \lambda$ is an isometric representation. A straightforward calculation reveals that $\Phi$ satisfies the cocycle identity with respect to $\lambda: \Phi(s t)=\lambda_{s}(\Phi(t))+\Phi(s)$ for all $s, t \in \Gamma$. Consequently, $\alpha_{s}=\lambda_{s}+\Phi(s)$ defines an affine isometric action of $\Gamma$ on $\bigoplus l^{p_{n}}(\Gamma)$. Observe that

$$
\|\Phi(s)-\Phi(t)\|=\left\|\alpha_{s}(0)-\alpha_{t}(0)\right\|=\left\|\alpha_{s^{-1} t}(0)\right\|=\left\|\Phi\left(s^{-1} t\right)\right\|,
$$

for all $s, t \in \Gamma$.

It remains only to check that $\Phi$ is proper, in the sense that $\|\Phi(s)\| \rightarrow \infty$ as $s \rightarrow \infty$ in $\Gamma$. This is straightforward. Indeed, if $f_{n}$ is supported in the ball of radius $R_{n}$ (in $\Gamma$, w.r.t. $|\cdot|$ ) and $s \in \Gamma$ satisfies $|s|>2 R_{n}$, then the supports of $s \cdot f_{n}$ and $f_{n}$ are disjoint and $\left\|s \cdot f_{n}-f_{n}\right\|_{p_{n}} \geq 1$ (since $\left\|f_{n}\right\|_{\infty}=1$ ). Hence, if $|s|>2 \max \left\{R_{1}, \ldots, R_{m}\right\}$, then $\|\Phi(s)\| \geq m^{1 / 2}$.

\section{REFERENCES}

[1] M. E. Bekka, P. A. Cherix, and A. Valette. Proper affine isometric actions of amenable groups. In Ferry et al. 2], pages 1-4. MR1388307 (97e:43001)

[2] S. Ferry, A. Ranicki, and J. Rosenberg, editors. Novikov Conjectures, Index Theorems and Rigidity. Number 226, 227 in London Mathematical Society Lecture Notes. Cambridge University Press, 1995. MR1388306 (96m:57003)

[3] M. Gromov. Asymptotic invariants of infinite groups. In A. Niblo and M. Roller, editors, Geometric Group Theory, number 182 in London Mathematical Society Lecture Notes, pages 1-295. Cambridge University Press, 1993. MR1253544|(95m:20041) 
[4] M. Gromov. Spaces and questions. Geom. Funct. Anal., Special Volume, Part I:118-161, 2000. GAFA 2000 (Tel Aviv, 1999). MR1826251 (2002e:53056)

[5] M. Gromov. Random walk in random groups. IHES preprint, 2002.

[6] E. Guentner, N. Higson, and S. Weinberger. The Novikov conjecture for linear groups. Preprint, 2003.

[7] E. Guentner and J. Kaminker. Exactness and the Novikov conjecture. Topology, 41(2):411418, 2002. MR1876896(2003e:46097a)

[8] N. Higson and E. Guentner. $K$-theory of group $C^{*}$-algebras. In S. Doplicher and R. Longo, editors, Noncommutative Geometry, Lecture Notes in Mathematics, pages 253-262. Springer Verlag, Berlin, 2003.

[9] G. Kasparov and G. Yu The coarse geometric Novikov conjecture and uniform convexity. Preprint.

[10] Jiří Matoušek. On embedding expanders into $l_{p}$ spaces. Israel J. Math., 102:189-197, 1997. MR.1489105(98k:46014)

[11] N. Ozawa. A note on non-amenability of $B\left(\ell_{p}\right)$ for $p=1,2$. Preprint.

[12] N. Ozawa. Amenable actions and exactness for discrete groups. C. R. Acad. Sci. Paris Sér. I Math., 330(8):691-695, 2000. MR1763912 (2001g:22007)

[13] G. Skandalis, J. L. Tu, and G. Yu. Coarse Baum-Connes conjecture and groupoids. Topology, 41:807-834, 2002. MR1905840 (2003c:58020)

[14] J. L. Tu. Remarks on Yu's Property A for discrete metric spaces and groups. Bull. Soc. Math. France, 129:115-139, 2001. Mr1871980(2002j:58038)

[15] G. Yu. The Coarse Baum-Connes conjecture for spaces which admit a uniform embedding into Hilbert space. Inventiones Math., 139:201-240, 2000. MR.1728880 (2000j:19005)

Department of Mathematics, Pennsylvania State University, University Park, PennSYLVANIA 16802

E-mail address: nbrown@math.psu.edu

Department of Mathematics, 2565 McCarthy Mall, University of Hawail, Mānoa, HonOlulu, HaWAil 96822-2273

E-mail address: erik@math.hawaii.edu 